\title{
PLASMONICS
}

\section{Backward waves moving forward}

\author{
The positive aspect of negative refraction is to open new possibilities for squeezing light into \\ tight spaces. Now, a metallic nanostructure pushes all-angle negative refraction into the \\ visible spectrum.
}

\section{Martin Wegener*, Gunnar Dolling and Stefan Linden \\ are at the DFG-Center for Functional Nanostructures (CFN), D-76128 Karlsruhe, Germany. *e-mail: martin.wegener@physik.uni-karlsruhe.de}

Y ou wake up, go into the bathroom and have a close look at your bathroom mirror. You may not have noticed, but the reflection you see is (partly) due to backward waves in the metal. Backward waves are an unusual kind of electromagnetic wave for which the phase velocity of light is opposite to the flow of electromagnetic energy. Such 'bathroom mirror physics' is closely related to a report in Science by Henri Lezec and colleagues who observe intriguing allangle negative refraction in the blue-green region of the visible spectrum ${ }^{1}$. However, to demonstrate such unusual effects, the researchers use a more complex structure than everyday bathroom mirrors, namely thin metal-dielectric-metal films that act as waveguides - a structure previously proposed from theory $y^{2,3}$.

Backward waves have recently attracted considerable interest in the context of photonic metamaterials ${ }^{4,5}$, where they form the basis for negative refraction ${ }^{6}$ and underlie so-called perfect lenses ${ }^{7,8}$, as well as 'hyperlenses ${ }^{99,10}$. All these concepts aim at achieving optical resolution well below half the wavelength of light - with obvious potential for optical imaging and lithography.

What makes backward waves so intriguing? Consider oblique incidence of light on a refractive medium. In a normal medium supporting forward waves, the incident beam of light experiences a positive beam displacement in transmission (Fig. 1, left panel). In contrast, a medium supporting backward waves leads to a negative displacement (Fig. 1, right panel). Any metal - including your bathroom mirror - supports backward waves parallel to the metal interface ${ }^{11}$ provided that the electric field has a non-zero component normal to the interface. Although not widely known, this behaviour is a simple
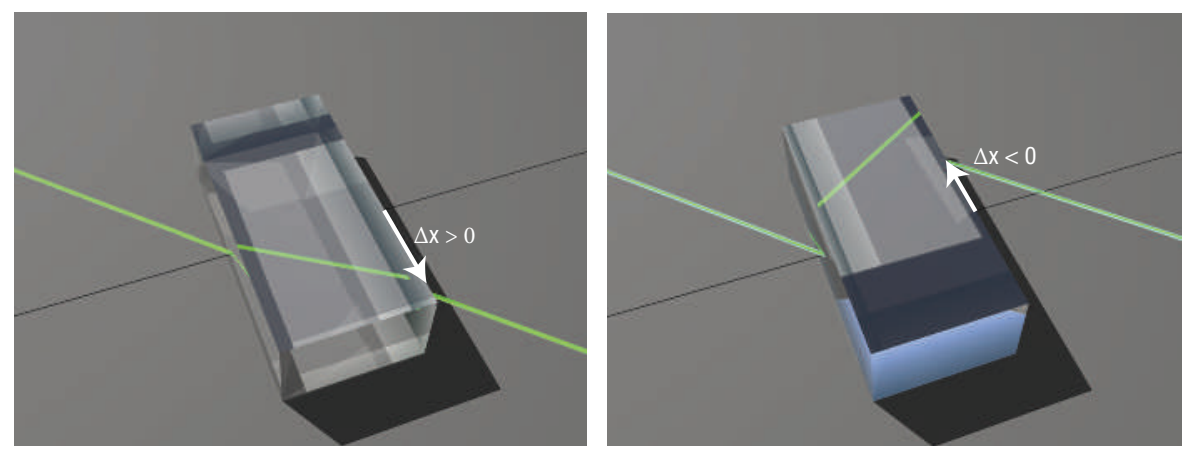

Figure 1 Positive versus negative refraction. In ray optics, a positive real part of the refractive index $(n>0)$ leads to a positive beam displacement $\Delta x$ (left panel), whereas the displacement is negative (right panel) for a negative refractive index $(n<0)$. In wave optics, however, the connection is not so simple. Even a single, thin (isotropic and homogeneous) metal film with $n>0$ can lead to negative displacements, hence to negative refraction.

and direct consequence of Maxwell's equations. For example, for a free-standing 25-nm-thin Ag film at 532-nm wavelength with $45^{\circ}$ angle of incidence of in-plane polarized light we obtain a negative lateral beam displacement of $\Delta x=-84 \mathrm{~nm}$ from exact analytical transfer-matrix calculations using literature permittivity values whereas the real part of the Ag refractive index $n$ is +0.05 and therefore positive.

This example illustrates that backward waves are ubiquitous in the context of metals. For surface-plasmon waves on a metal-dielectric interface, however, the electromagnetic wave is backward only inside the metal but forward inside the dielectric, where the larger fraction of the energy flows. Thus, usually, the net behaviour is a forward wave. In contrast, for the metal-dielectric-metal $\left(\mathrm{Au} / \mathrm{Si}_{3} \mathrm{~N}_{4} / \mathrm{Ag}\right)$ waveguide shown in Fig. $2 \mathrm{a}$ and used by Lezec and colleagues ${ }^{1}$, the wave is pushed into the metal by restricting the $\mathrm{Si}_{3} \mathrm{~N}_{4}$ layer to a subwavelength thickness of $t_{1}=50 \mathrm{~nm}$ and, hence, a net backward-wave behaviour results ${ }^{2}$. Unfortunately, this wave turns out to be very strongly damped ${ }^{1}$ with an absorption length of about $50 \mathrm{~nm}$. This means that the intensity is reduced by more than four orders of magnitude after propagation over just half a micrometre in distance. Lezec and colleagues have solved the resulting measurement problem by coupling light in and out of the structure by using 'slits' at specific positions (see 'Input' and 'Output' in Fig. 2a) located on opposite sides. Furthermore, they used an additional auxiliary waveguide with much larger dielectric spacer thickness $t_{2}=500 \mathrm{~nm}$ that supports the incident light and transmits net forward waves. In this fashion, they were able to demonstrate negative refraction by using several variants of Fig. 1, for example, by the prism geometry depicted in Fig. 2b.

Negative refraction in the visible region has previously been demonstrated experimentally for both dielectric photonic crystals ${ }^{12,13}$ and dielectric birefringent materials ${ }^{14}$. Broadly speaking, however, one has to carefully differentiate between the two notions 'negative refraction' and 'negative refractive index'. Even for the above example of a single (isotropic and homogeneous) thin metal film, negative refraction in the sense of Fig. 1 is possible while the refractive index $n$ remains positive. Thus, in wave optics there is no simple general relation between the sign of refraction and the sign of the refractive index $n$. In contrast, in ray 

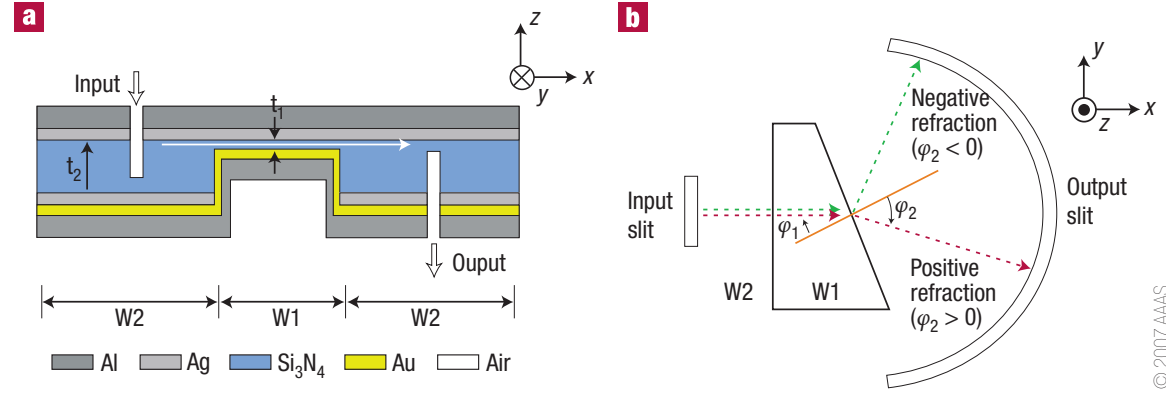

Figure 2 Plasmonic waveguide structure. Illustration of the plasmonic nanostructure used by Henri Lezec and co-workers for the demonstration of all-angle negative refraction in the visible region'. The W1 regions support backward waves for green light (forward waves for red light), the W2 regions support forward waves. a, Side view and $\mathbf{b}$, Top view. Reprinted in part with permission from ref. 1.

optics the sign of the beam displacement in Fig. 1 is strictly identical to that of the refractive index $n$. The logical conclusion is that we should stop calling the refractive index $n$ 'refractive index'. We should rather call it the 'slowness factor' according to its definition: The phase velocity of light $c$ is slower by factor $n$ inside the material than the vacuum speed of light $c_{0}$.

Backward waves may seem strange but they are certainly much less rare a species than it is tempting to believe at first sight. In the visible, damped backward waves occur in your bathroom mirror, reduced-loss backward waves in related metal-dielectric-metal waveguide structures $^{1-3}$, as well as in conceptually distinct photonic metamaterials $\mathrm{s}^{4,5}$. As Lezec and colleagues demonstrate, such tailored metallic nanostructures provide us with many new opportunities for optics and photonics applications that natural optical substances just do not have. More surprises can be expected in future.

\section{References}

1. Lezec, H. J., Dionne, J. A. \& Atwater, H. A. Science 316, 430-432 (2007).

2. Tournois, P. \& Laude, V. Opt. Commun 137, 41-45 (1997).

3. Shin, H. \& Fan, S. Phys. Rev. Lett. 96, 073907 (2006).

4. Soukoulis, C. M., Linden, S. \& Wegener, M. Science 315, 47-49 (2007).

5. Shalaev, V. Nature Photon. 1, 41-48 (2006).

6. Shelby, R. A., Smith, D. R. \& Schultz, S. Science 292, 77-79 (2001)

7. Pendry, J. B. Phys. Rev. Lett. 85, 3966-3969 (2000).

8. Fang, N., Lee, H., Sun, C. \& Zhang, X. Science 308, 534-537 (2005).

9. Liu, Z., Lee, H., Xiong, Y., Sun, C. \& Zhang, X. Science 315, 1686 (2007)

10. Smolyaninov, I. I., Hung, Y.-J. \& Davis, C. C. Science 315, 1699-1701 (2007).

11. Lai, H. M., Kwok, C. W., Loo, Y. W. \& Xu, B. Y. Phys. Rev. E 62, 7330-7339 (2000)

12. Russell, P. St. J. Phys. Rev. A 33, 3232-3242 (1986).

13. Zengerle, R. J. Mod. Opt. 34, 1589-1617 (1987).

14. Zhang, Y., Fluegel, B. \& Mascarenhas, A. Phys. Rev. Lett. 91, 157404 (2003).

\section{MATERIAL WITNESS}

\section{Tendentious tilings}

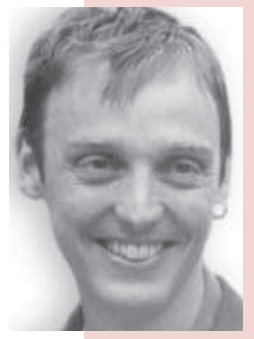

Quasicrystal enthusiasts may have been baffled by a rather cryptic spate of comments and clarifications following in the wake of a recent article claiming that medieval Islamic artists had the tools needed to construct quasicrystalline patterns. That suggestion was made by Peter Lu at Harvard University and Paul Steinhardt at Princeton (Science 315, 1106-1110; 2007). But in a news article in the same issue, staff writer John Bohannon explained that these claims had already caused controversy, being allegedly anticipated in the work of crystallographer Emil Makovicky at the University of Copenhagen (Science 315,1066 ; 2007).

The central thesis of Lu and Steinhardt is that Islamic artists used a series of tile shapes, which they call girih tiles, to construct their complex patterns. They can be used to make patterns of interlocking pentagons and decagons with the 'forbidden' symmetries characteristic of quasicrystalline metal alloys, in which these apparent symmetries, evident in diffraction patterns, are permitted by a lack of true periodicity.
Although nearly all of the designs evident on Islamic buildings of this time are periodic, Lu and Steinhardt found that those on a fifteenth-century shrine in modernday Iran can be mapped almost perfectly onto another tiling scheme, devised by mathematician Roger Penrose, which does generate true quasicrystals.

But in 1992 Makovicky made a very similar claim for a different Islamic tomb dating from 1197. Some accused Lu and Steinhardt of citing Makovicky's work in a way that did not make this clear. The authors, meanwhile, admitted that they were unconvinced by Makovicky's analysis and didn't want to get into an argument about it.

The dispute has ruffled feathers. Science subsequently published a 'clarification' that irons out barely perceptible wrinkles in Bohannon's article, while Lu and Steinhardt attempted to calm the waters with a letter in which they 'gladly acknowledge' earlier work (Science 316, 982; 2007). It remains to be seen whether that will do the trick, for Makovicky wasn't the only one upset by their paper. Design consultant Jay Bonner in Santa Fe has also made previous links between Islamic patterns and quasicrystals (see http://www.bonner-design.com/ publications/self-similar.htm).
Most provocatively, Bonner discusses the late-fifteenth-century Topkapi architectural scroll that furnishes the key evidence for Lu and Steinhardt's girih scheme. Bonner points out how this scroll reveals explicitly the "underlying polygonal sub-grid" used to construct the pattern it depicts. He proposes that the artists commonly used such a polygonal matrix, composed of tile-like elements, and demonstrates how these can create aperiodic space-filling designs.

Bonner does not mention quasicrystals, and his use of terms such as self-similarity and even symmetry do not always fit easily with that of physicists and mathematicians. But there's no doubting that his work deepens the "can of worms" that Bohannon says Lu and Steinhardt have opened.

All this suggests that the satellite conference of the forthcoming European Crystallographic Meeting in Marrakech this August, entitled "The enchanting crystallography of Moroccan ornaments", might be more stormy than enchanting - for it includes back-to-back talks by Makovicky and Bonner.

Philip Ball 